This information is current as of April 25, 2023. 


\title{
Morphologic Characteristics of Atherosclerotic Middle Cerebral Arteries on 3T High-Resolution MRI
}

\author{
X.J. Zhu, B. Du, X. Lou, F.K. Hui, L. Ma, B.W. Zheng, M. Jin, C.X. Wang, and W.-J. Jiang
}

\section{ABSTRACT}

BACKGROUND AND PURPOSE: There are limited studies on the morphologic characteristics of MCA atherosclerotic stenosis. Our aim was to quantitatively assess the remodeling pattern and plaque distribution of atherosclerotic MCAs with $3 T$ high-resolution MR imaging.

MATERIALS AND METHODS: Eighty-seven consecutive patients with symptomatic atherosclerotic stenoses at the Ml segment of the MCA on DSA (50\%-99\%) were enrolled. The remodeling index was calculated as the Vessel Area at Maximal Lumen Narrowing/Reference Vessel Area. A remodeling index $\geq 1.0$ was defined as positive remodeling, and a remodeling index $<1.0$, as negative remodeling. Plaque distribution at the maximal lumen narrowing site was classified on the basis of the involvement of the superior, inferior, dorsal, or ventral MCA wall.

RESULTS: Forty-three of 87 patients were excluded due to poor imaging quality $(n=8)$ or scan plane obliquity secondary to a tortuous M1 segment of the MCA or an MCA ostium lesion or angled lesion $(n=35)$. Of 44 patients in the final analysis, negative remodeling was found in 19 (43.2\%) lesions, and positive remodeling, in 25 (56.8\%) lesions. At maximal lumen narrowing sites, lesions with negative remodeling had less vessel area, wall area, and percentage of plaque burden $(P<.0001)$ and a lower eccentricity index $(P=.023)$, compared with lesions with positive remodeling. The plaque involved the superior and dorsal walls in $15(34.1 \%)$ of 44 patients.

CONCLUSIONS: 2D high-resolution MR imaging can help assess the remodeling pattern and plaque distribution of MCA stenosis, but the imaging and postprocessing protocol for remodeling assessment needs to be improved in the tortuous course of the MCA and in MCA ostium or angled lesions.

ABBREVIATIONS: $M L N=$ maximal lumen narrowing; $N R=$ negative remodeling; PR = positive remodeling; PTAS = percutaneous transluminal angioplasty and stenting; SAMMPRIS = Stenting versus Aggressive Medical Management for Preventing Recurrent Stroke in Intracranial Arterial Stenosis

$\mathbf{P}$ revious studies of coronary arteries confirmed that vessels may respond to plaque growth by either outward enlargement of the vessel wall (positive remodeling) or vessel shrinkage (negative re-

Received July 9, 2012; accepted after revision October 29.

From the Department of Neurology (X.Z., B.Z., C.W.), Beijing Tiantan Hospital, Capital Medical University, Beijing, China; New Era Stroke Care and Research Institute (B.D., M.J., W.-J.J.), The Second Artillery General Hospital People's Liberation Army, Beijing, China; Department of Radiology (X.L., L.M.), Chinese People's Liberation Army General Hospital, Beijing, China; and Cerebrovascular Center (F.K.H.), Cleveland Clinic Foundation, Cleveland, Ohio.

All the images were assessed and measured by X.Z. (reader 1) and B.Z. (reader 2). Wei-Jian Jiang is supported by grants from the National Basic Research Program (program 973) of China (2013CB733805) and the National Natural Science Foundation of China (30670731, 81070925). Chunxue Wang is supported by grants from the National Science Foundation (81071115). Xin Lou is supported by grants from the National Natural Science Foundation of China (81101034).

Please address correspondence to Wei-Jian Jiang, MD, New Era Stroke Care and Research Institute, The Second Artillery General Hospital PLA; 16 Xinjiekouwai Ave, Beijing 100088, China; e-mail cjr.jiangweijian@vip.163.com; or Chunxue Wang, MD, Department of Neurology, Beijing Tiantan Hospital, Capital Medical University, 6 Tiantan Xili, Dongcheng District, Beijing 100050; China; e-mail chunxue.sen@gmail.com

- Indicates open access to non-subscribers at www.ajnr.org

http://dx.doi.org/10.3174/ajnr.A3573 modeling). ${ }^{1}$ Pre-existing remodeling was important in the immediate success and clinical outcome following coronary interventions. ${ }^{2,3}$ In addition, the existence of plaque close to a branch vessel ostium has been shown to increase the risk of branch occlusion after coronary stent placement. ${ }^{4}$ In light of the disappointing results ${ }^{5}$ of the Stenting versus Aggressive Medical Management for Preventing Recurrent Stroke in Intracranial Arterial Stenosis (SAMMPRIS) trial, we are reminded that lesion morphology of the intracranial artery may also be a prominent factor that influences the rate of periprocedural complications. ${ }^{6,7}$ Knowledge of the wall characteristics of the MCA may be useful in interpreting the data of the SAMMPRIS trial, selecting patients for percutaneous transluminal angioplasty and stenting (PTAS), and designing individualized operating plans.

High-resolution MR imaging has been increasingly used in recent years to identify the wall features of the intracranial artery. ${ }^{8-12}$ However, the remodeling pattern and the plaque distribution of MCA atherosclerotic stenosis remains poorly understood. The aim of this prospective study was to quantitatively investigate remodeling patterns and plaque distribution in patients with symptomatic MCA stenosis with $3 \mathrm{~T}$ high-resolution MR imaging. 

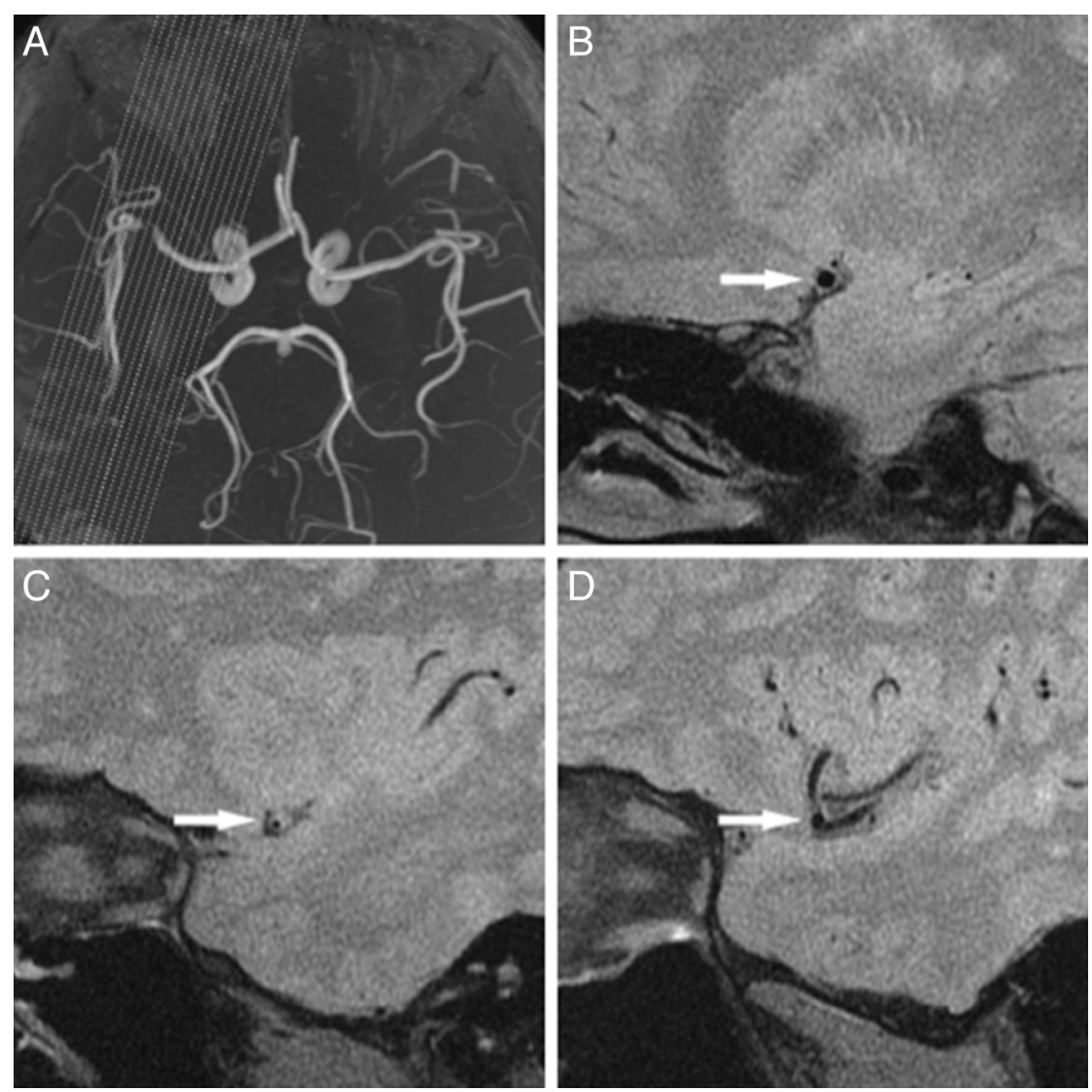

FIG 1. An example of an excluded case. MRA $(A)$ shows the obvious angled stenosis located at the distal M1 segment near M2 of the MCA. Suitable cross-sectional images can be obtained at the proximal (B, arrow) and MLN (C, arrow) but not the distal ( $D$, arrow) site.

\section{MATERIALS AND METHODS \\ Patients}

This observational cross-sectional study was approved by our institutional ethics committees. Before each high-resolution MR imaging examination, written informed consent was obtained. Patients were enrolled in this study according to the following criteria: 1) ischemic stroke or TIA in the target MCA territory within 90 days; 2) 50\%-99\% stenosis at the M1 segment of the MCA on DSA; and 3) $\geq 2$ atherosclerotic risk factors, including hypertension, hypercholesterolemia, diabetes mellitus, cigarette smoking, and obesity as described previously. ${ }^{10}$ According to the traditional clinical definition, we defined ischemic stroke as a new focal neurologic deficit of sudden onset lasting $>24$ hours and not caused by hemorrhage, and TIA is the acute onset of a focal neurologic deficit lasting $<24$ hours. We excluded patients with the following conditions: 1) contraindications to MR imaging; 2) nonatherosclerotic vasculopathy, such as dissection, arteritis, or Moyamoya disease; 3 ) fewer than 2 atherosclerotic risk factors; 4) coexistent $>50 \%$ supra-aortic cerebrovasculature stenosis on DSA; and 5) evidence of cardioembolism.

Between December 2009 and May 2011, eighty-seven consecutive patients with symptomatic atherosclerotic stenosis at the M1 segment of the MCA on DSA (50\%-99\%) were enrolled in this study.

\section{Imaging Protocol}

Cross-sectional imaging was performed by using a 3T MR imaging scanner (Signa, TwinSpeed 3T; GE Healthcare, Milwaukee,
Wisconsin) and an 8-channel phased array head coil. 3D time-of-flight MRA was performed purely for image positioning with the following parameters: TR/TE = $21 / 3.2 \mathrm{~ms}, \mathrm{FOV}=16 \times 16 \mathrm{~cm}$, thickness $=1 \mathrm{~mm}$, matrix $=256 \times 256$, and $\mathrm{NEX}=1$. On the basis of the MRA, the scan plane was angled to ensure that the cross-sectional images were perpendicular to the M1 segment of the MCA (Figs 1A, 2A, and $3 A$ ). Then, proton attenuationweighted images of 12-17 sections were acquired with an FSE sequence. The parameters for proton attenuation-weighted images were as follows: TR/TE $=4000 / 12.9$ $\mathrm{ms}, \mathrm{FOV}=16 \times 16 \mathrm{~cm}$, thickness $=2 \mathrm{~mm}$, matrix $=384 \times 256$, NEX $=2$, and echotrain length $=10$. The cross-sectional voxel size was $0.6 \times 0.6 \mathrm{~mm}$ on time-of-flight images and $0.4 \times 0.6 \mathrm{~mm}$ on proton attenuation-weighted images. Fat suppression was applied to reduce fat signal from the surrounding scalp. A zero-fill interpolation 512-matrix technique was used to enhance spatial resolution. The total imaging time was 7-10 minutes.

\section{Morphologic Measurement and Calculation of the MCA Wall}

Two trained readers with $>1$ year of experience in reading intracranial MR images, blinded to the clinical information of the patients, assessed image quality by consensus by using a previously developed 4 -point scale ( 1 = poor quality, $4=$ excellent). ${ }^{13}$ Images of poor image quality were excluded from the final analysis. Quantitative measurement was performed on the proton attenuationweighted images with a score of $\geq 2$ by using FuncTool II software (GE Healthcare) from a Sun ADW4.3 workstation (GE Healthcare). The images were zoomed to $400 \%$. The window width and level were adjusted to optimize the conspicuity of the vessel contour. In the MCA cross-sectional images, the contour of the outer wall and lumen at the maximal lumen narrowing site and reference site was traced manually to measure the vessel area and luminal area by reader 1 , blinded to the clinical information of the patients. The reference sites used were the nearest plaque-free or minimally diseased segments proximal and distal to the maximal lumen narrowing (MLN) sites.

Due to the vessel tapering, the reference vessel area was calculated as the average of the distal and proximal vessel area, and luminal area, was calculated as the average of the distal and proximal luminal area. Wall area was calculated by Vessel Area - Luminal Area. Plaque size was estimated by Wall Area at the MLN site - Reference Wall Area. Because plaque size varies with vessel size and larger vessels usually have larger plaques, we used the percentage of plaque burden to correct for vessel sizes. The percentage of plaque burden was calculated by using the following formula: (Plaque Size/Vessel Area at MLN site) $\times 100 \%$. The degree of stenosis on high-resolution MR imaging 

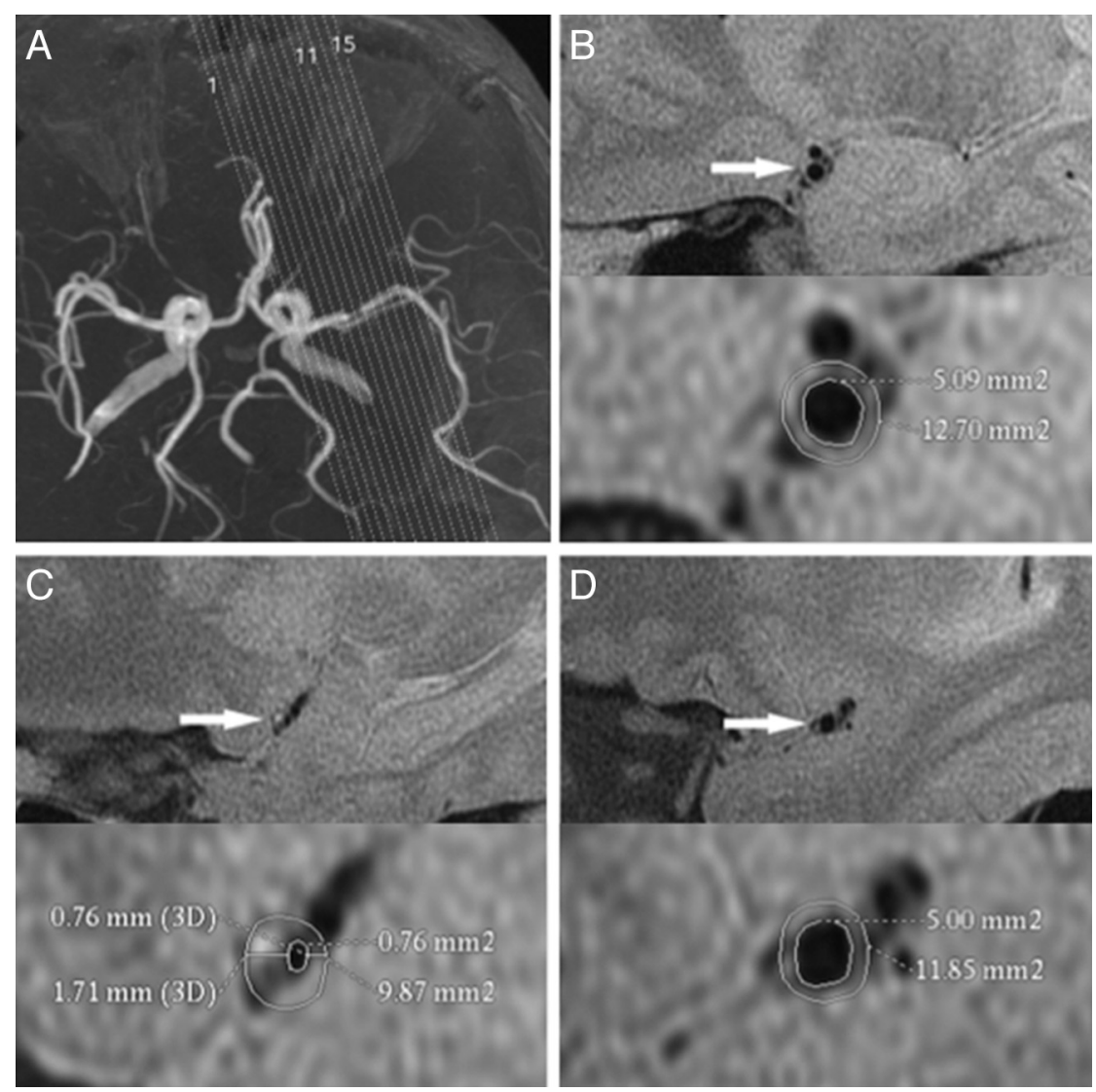

FIG 2. A 37-year-old man with hypertension, hypercholesterolemia, and cigarette smoking history who presented with aphasia, a cognitive disorder, and right-side numbness for 60 days. MRA ( $A$ ) shows stenosis located at the straight M1 segment of the MCA. The proximal ( $B$, arrow), $\operatorname{MLN}(C$, arrow), and distal $(D$, arrow) site of the left MCA are shown. The vessel area or luminal area is measured (demonstrated by the magnified view in the inset). The reference vessel area is $12.3 \mathrm{~mm}^{2}$, and the remodeling index at the MLN site is $0.8<1.00$ (NR). The eccentricity index is 0.6 . $B$, The plaque location is mainly on the ventral wall (arrow). The wall area is $7.6 \mathrm{~mm}^{2}$ at the proximal, $9.1 \mathrm{~mm}^{2}$ at the MLN, and $6.9 \mathrm{~mm}^{2}$ at the distal site. The reference wall area is $7.2 \mathrm{~mm}^{2}$. Plaque size is $1.9 \mathrm{~mm}^{2}$, and percentage of plaque burden is $19.1 \%$.

was calculated as follows: (1 - Luminal Area at the MLN Site/Reference Luminal Area) $\times 100 \%{ }^{1}$ The remodeling index was calculated as follows: Vessel Area at MLN Site/Reference Vessel Area. Positive remodeling $(\mathrm{PR})$ was defined as a remodeling index $\geq 1.0$; negative remodeling $(\mathrm{NR})$, as a remodeling $<1.0 .{ }^{1}$ Patterns of the remodeling were classified into 2 subgroups (PR and NR).

The thickest and thinnest wall segments on the cross-sectional image at the MLN site were identified by visual inspection, and maximal and minimal wall thicknesses were measured manually by the same reader (reader 1). The eccentricity index of the MLN site was calculated as follows: (Maximal Wall Thickness - Minimal Wall Thickness)/Maximal Wall Thickness. ${ }^{14}$ The maximal wall thickness at the reference site was the average of maximal wall thickness distal and proximal to the MLN site. All cross-sectional images at MLN sites were classified on the basis of their plaque orientation being centered on the superior, inferior, dorsal, or ventral side of the vessel. ${ }^{15}$ Each cross-sectional image at the MLN site was grouped into 1 of the 4 quadrants. In cases in which the plaque was distributed on $>1$ quadrant, the quadrant with the maximal wall thickness was chosen.

To assess interobserver variability and intraobserver variability, the 2 readers independently remeasured the vessel area, lumi- nal area, maximal wall thickness, and minimal wall thickness at MLN sites of the initial 10 consecutive patients 2 months later. Subjects, included 2 patients (score 2), 6 patients (score 3 ), and 2 patients (score 4).

\section{Statistical Analysis}

The intraclass correlation coefficient was used to find the intraobserver or interobserver reproducibility for the measurements of vessel area, luminal area, maximal wall thickness, and minimal wall thickness. Continuous variables were described as means \pm SD or as an interquartile range (if not normally distributed). The continuous variable between the 2 groups was compared by using the Student $t$ test or Mann-Whitney $U$ test (when the continuous variable was not normally distributed). Categoric variables were compared by using the $\chi^{2}$ test or Fisher exact test. The Statistical Package for the Social Sciences, Version 11.5 (SPSS, Chicago, Illinois) was used as the statistical analysis software. All reported $P$ values were 2 -sided, and a $P$ value $<.05$ was considered statistically significant.

\section{RESULTS \\ Patients}

From the 87 patients enrolled in this study, 8 (9.2\%) were excluded due to poor image quality and $79(90.8 \%)$ had images suitable for analysis (image quality, $\geq 2$ ) to depict the MCA wall. In the final analysis, 35 patients were also excluded because cross-sectional images perpendicular to the MCA at reference sites $(n=34)$ or at the MLN site $(n=1)$ could not be acquired due to a tortuous course of the MCA or a lesion at the MCA ostia or an angled lesion (Fig 1). Therefore, 44 patients ( 39 men and 5 women) were included in the final analysis. There were no significant differences in the clinical characteristics between the included patients and excluded patients (Table 1). The mean age of included patients was $47.8 \pm$ 9.9 years. The image quality was 2 in 8 patients, 3 in 26 patients, and 4 in 10 patients. The mean time from qualifying events to high-resolution MR imaging was $33.8 \pm 19.0$ days.

\section{Morphologic Measurement and Calculation of the MCA Wall}

The intraobserver and interobserver reproducibility was high for measurements of the vessel area, luminal area, maximal wall thickness, and minimal wall thickness (Table 2).

At the reference site, the vessel area was $14.8 \pm 3.3 \mathrm{~mm}^{2}$, the luminal area was $5.3 \pm 1.8 \mathrm{~mm}^{2}$, the wall area was $9.5 \pm 1.7 \mathrm{~mm}^{2}$, and the maximal wall thickness was $0.9 \pm 0.1 \mathrm{~mm}$. At the MLN site, the vessel area was $15.4 \pm 4.8 \mathrm{~mm}^{2}$, the luminal area was $0.8 \pm 0.6 \mathrm{~mm}^{2}$, and the wall area was $14.6 \pm 4.7 \mathrm{~mm}^{2}$. Thus, 

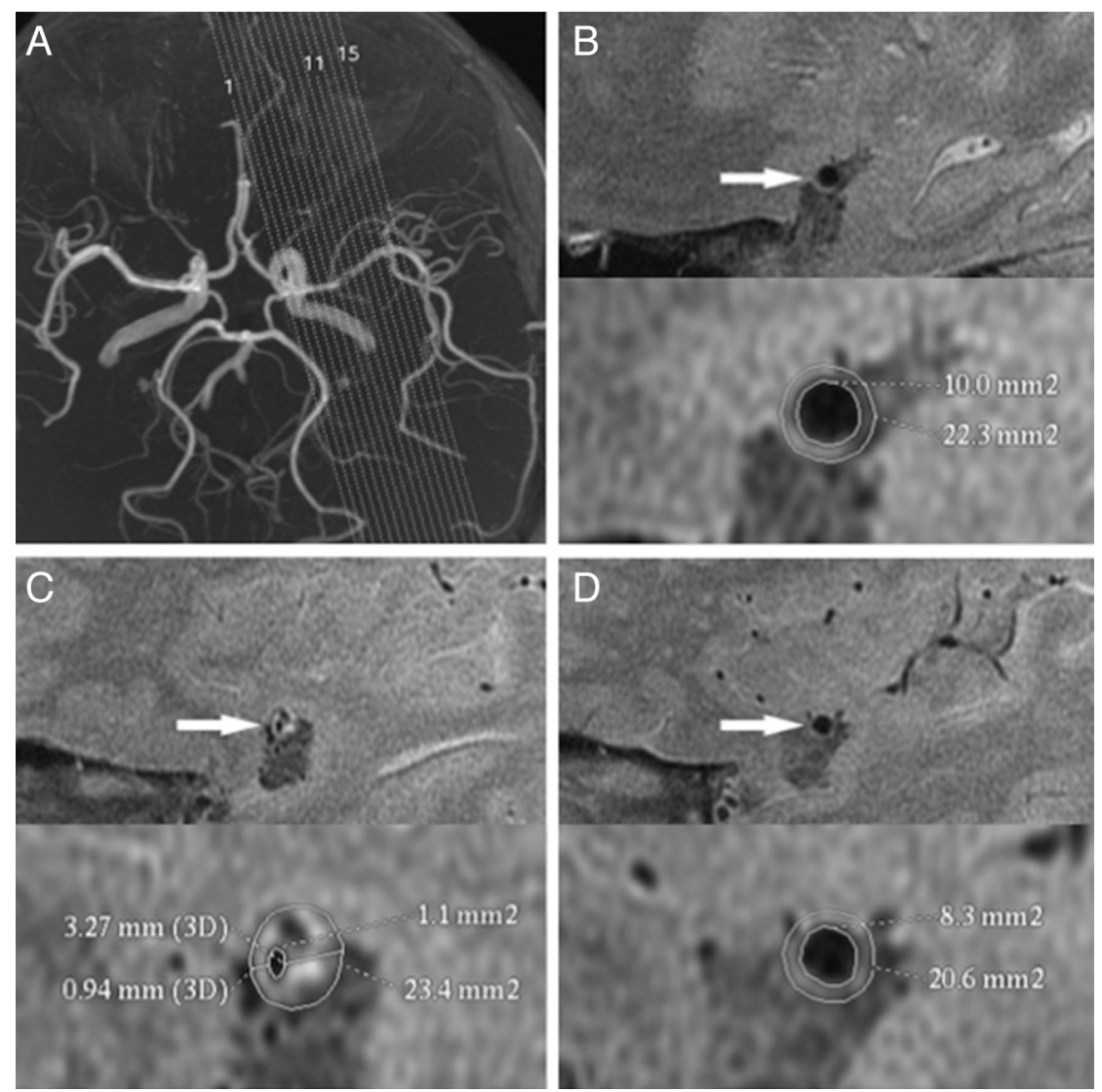

FIG 3. A 74-year-old man with hypertension and hypercholesterolemia who presented with aphasia for 27 days. MRA (A) shows stenosis located at the nearly straight Ml segment of the MCA. The proximal ( $B$, arrow), MLN (C, arrow), and distal (D, arrow) site of left MCA are surrounded by more CSF due to age-related brain involution compared with the case in Fig 2 . The vessel area or lumen area is measured (demonstrated by the magnified view in the inset). The reference vessel area is $21.5 \mathrm{~mm}^{2}$, and the remodeling index at the MLN is $1.1>1.00$ (PR). The eccentricity index is 0.7 . $B$, The plaque location is mainly on the ventral and superior walls (arrow). The wall area is $12.3 \mathrm{~mm}^{2}$ at the proximal, $22.3 \mathrm{~mm}^{2}$ at the $\mathrm{MLN}$, and $12.3 \mathrm{~mm}^{2}$ at the distal site. The reference wall area is $12.3 \mathrm{~mm}^{2}$. Plaque size is $10.0 \mathrm{~mm}^{2}$, and the percentage of plaque burden is $42.7 \%$. Compared with the case in Fig 2, this case has a larger vessel area, wall area, plaque size, percentage of plaque burden, and eccentricity index.

Table 1: Clinical characteristics between included patients and excluded patients

\begin{tabular}{lccc}
\hline \multicolumn{1}{c}{ Characteristics } & $\begin{array}{c}\text { Included } \\
\text { Patients } \\
(\boldsymbol{n}=\mathbf{4 4 )}\end{array}$ & $\begin{array}{c}\text { Excluded } \\
\text { Patients } \\
(\boldsymbol{n}=\mathbf{4 3})\end{array}$ & $\begin{array}{c}\boldsymbol{P} \\
\text { Value }\end{array}$ \\
\hline Age (yr) (mean) (SD) & $47.8(9.9)$ & $47.7(11.4)$ & .966 \\
Men (No.) (\%) & $39(88.6)$ & $37(86.1)$ & .716 \\
Hypertension (No.) (\%) & $29(65.9)$ & $26(60.5)$ & .599 \\
Hypercholesterolemia (No.) (\%) & $42(95.5)$ & $42(97.7)$ & 1.000 \\
Diabetes mellitus (No.) (\%) & $8(18.2)$ & $10(23.3)$ & .559 \\
Smoking (No.) (\%) & $30(68.2)$ & $31(72.1)$ & .690 \\
Obesity (No.) (\%) & $7(15.9)$ & $3(7.0)$ & .332 \\
Three or more risk factors (No.) (\%) & $23(52.3)$ & $22(51.2)$ & .918 \\
Stroke as qualifying event (No.) (\%) & $27(61.4)$ & $26(60.5)$ & .932 \\
NiHSS scores at admission & $0.0(0.0-1.0)$ & $0.0(0.0-1.0)$ & .916 \\
$\quad$ (median) (interquartile range) & & & \\
Time from qualifying event to & $33.8(19.0)$ & $33.3(21.2)$ & .905 \\
$\quad$ high-resolution MRI, days & & & \\
$\quad$ (mean) (SD) & & & \\
\hline
\end{tabular}

plaque size was $5.1 \pm 4.5 \mathrm{~mm}^{2}$, percentage of plaque burden was $28.1 \pm 21.1 \%$, degree of stenosis was $82.8 \pm 14.2 \%$, and remodeling index was $1.1 \pm 0.3$. At the MLN sites, the maximal wall thickness was $2.5 \pm 0.6 \mathrm{~mm}$, the minimal wall thickness was $1.0 \pm 0.3 \mathrm{~mm}$, and the eccentricity index was $0.6 \pm 0.2$.

NR was found in 19 (43.2\%) lesions (Fig 2), and PR, in 25 (56.8\%) lesions (Fig 3). There was no significant difference in the clinical characteristics between the lesions with NR and PR (Table 3). The wall morphologic characteristics are outlined in Table 4. At MLN sites, lesions with NR, compared with lesions with $\mathrm{PR}$, had less vessel area, wall area, plaque size, and percentage of plaque burden $(P<.0001)$. At the same time, lesions with NR had a significantly lower maximal wall thickness $(P<.0001)$ and eccentricity index than did lesions with PR ( 0.5 versus $0.6, P=$ .023). However, there was no difference in the luminal area, minimal wall thickness, and degree of stenosis between the lesions with NR and PR. At reference sites, NR lesions had greater maximal wall thickness than PR lesions, with a trend toward significance; however, there was no significant difference in vessel area, luminal area, and wall area between the 2 groups.

Plaques were located at the superior wall in $10(22.7 \%)$ patients, the dorsal wall in $5(11.4 \%)$ patients, the inferior wall in 13 (29.6\%) patients, and the ventral wall in 16 (36.4\%) patients (Fig 2). In total, the plaque involved the superior and dorsal walls in $34.1 \%$ of patients (Fig 3).

\section{DISCUSSION}

The use of high-resolution MR imaging could permit evaluation of the remodeling of intracranial arteries. ${ }^{8-12}$ A previous study showed that the remodeling index could be assessed quantitatively with $1.5 \mathrm{~T}$ high-resolution MR imaging. ${ }^{8}$ Compared with $1.5 \mathrm{~T}$, a 3T MR imaging system has higher SNR and has recently been used to identify the remodeling pattern of intracranial arteries. ${ }^{10,11}$ The results showed that NR and PR also occur in the MCA and basilar arteries. ${ }^{10,11}$ However, the remodeling index depends on the reference vessel area. Using only proximal or distal segments as a reference site in previous studies may have resulted in underestimation or overestimation of the remodeling index due to the natural tapering of the MCA. ${ }^{11}$ In contrast, we used the average of reference site areas distal and proximal to the stenosis for remodeling calculation to help overcome the obstacle of natural tapering of the MCA. In addition, the positioning of $2 \mathrm{D}$ sections in 1 scan for the tortuous MCA makes 2D images more prone to obliquity artifacts, especially for the reference sites, leading to the overestimation of the true vessel area and wall thickness. To minimize errors, we excluded 35 patients because cross-sectional images perpendicular to the long axis of the MCA were unavailable. 
Table 2: Intraobserver and interobserver variability

Intraclass Correlation Coefficient

$(95 \% \mathrm{Cl})$

\begin{tabular}{lcc}
\cline { 2 - 3 } & Intraobserver & Interobserver \\
\hline Vessel area & $0.917(0.666-0.979)$ & $0.906(0.620-0.977)$ \\
Luminal area & $0.833(0.329-0.959)$ & $0.898(0.589-0.975)$ \\
Maximal wall thickness & $0.962(0.847-0.991)$ & $0.916(0.663-0.979)$ \\
Minimal wall thickness & $0.876(0.501-0.969)$ & $0.851(0.399-0.963)$ \\
\hline
\end{tabular}

Table 3: Clinical characteristics between the NR and PR groups

\begin{tabular}{lccr}
\hline \multicolumn{1}{c}{ Characteristics } & $\begin{array}{c}\text { NR Group } \\
(\boldsymbol{n}=\mathbf{1 9})\end{array}$ & $\begin{array}{c}\text { PR Group } \\
(\boldsymbol{n}=\mathbf{2 5})\end{array}$ & $\begin{array}{c}\boldsymbol{P} \\
\text { Value }\end{array}$ \\
\hline Age (yr) (mean) (SD) & $48.0(8.4)$ & $47.8(11.0)$ & .951 \\
Men (No.) (\%) & $15(79.0)$ & $24(96.0)$ & .198 \\
Hypertension (No.) (\%) & $13(68.4)$ & $16(64.0)$ & .759 \\
Hypercholesterolemia (No.) (\%) & $19(100)$ & $23(92.0)$ & .498 \\
Diabetes mellitus (No.) (\%) & $5(26.3)$ & $3(12.0)$ & .409 \\
Smoking (No.) (\%) & $11(57.9)$ & $19(76.0)$ & .202 \\
Obesity (No.) (\%) & $5(26.3)$ & $2(8.0)$ & .219 \\
Three or more risk factors (No.) (\%) & $12(63.2)$ & $11(44.0)$ & .208 \\
Stroke as qualifying event (No.) (\%) & $13(68.4)$ & $14(56.0)$ & .402 \\
NIHSS scores at admission, & $0.0(0.0-2.0)$ & $0.0(0.0-0.5)$ & .419 \\
$\quad$ median (interquartile range) & & & \\
Time from qualifying event to & $33.8(17.4)$ & $33.8(20.5)$ & 1.000 \\
$\quad$ high-resolution MRI (days) & & & \\
(mean) (SD) & & & \\
\hline
\end{tabular}

Table 4: Wall characteristics of symptomatic atherosclerotic MCA stenosis

\begin{tabular}{|c|c|c|c|}
\hline Variables $^{\mathrm{a}}$ & $\begin{array}{c}\text { NR Group } \\
(n=19)\end{array}$ & $\begin{array}{c}\text { PR Group } \\
(n=25)\end{array}$ & $\begin{array}{c}P \\
\text { Value }\end{array}$ \\
\hline \multicolumn{4}{|l|}{ At reference site } \\
\hline Vessel area $\left(\mathrm{mm}^{2}\right)$ & $15.5(3.3)$ & $14.3(3.2)$ & .228 \\
\hline Luminal area $\left(\mathrm{mm}^{2}\right)$ & $5.5(1.6)$ & $5.2(1.9)$ & .516 \\
\hline Wall area $\left(\mathrm{mm}^{2}\right)$ & $10.0(1.8)$ & $9.1(1.6)$ & .103 \\
\hline Maximal wall thickness (mm) & $1.0(0.1)$ & $0.9(0.1)$ & .058 \\
\hline \multicolumn{4}{|l|}{ At MLN site } \\
\hline Vessel area $\left(\mathrm{mm}^{2}\right)$ & $12.4(3.3)$ & $17.8(4.5)$ & $<.0001$ \\
\hline Luminal area $\left(\mathrm{mm}^{2}\right)$ & $0.8(0.6)$ & $0.8(0.5)$ & 0.804 \\
\hline Wall area $\left(\mathrm{mm}^{2}\right)$ & $11.6(3.3)$ & $16.9(4.3)$ & $<.0001$ \\
\hline Plaque size $\left(\mathrm{mm}^{2}\right)$ & $1.6(2.3)$ & $7.8(3.8)$ & $<.0001$ \\
\hline Percentage of plaque burden (\%) & $9.9(16.8)$ & $42.0(11.1)$ & $<.0001$ \\
\hline Degree of stenosis (\%) & $84.5(13.8)$ & $81.5(14.6)$ & .497 \\
\hline Maximal wall thickness (mm) & $2.1(0.5)$ & $2.8(0.5)$ & $<.0001$ \\
\hline Minimal wall thickness, (mm) & $0.9(0.3)$ & $1.0(0.2)$ & .381 \\
\hline Eccentricity index & $0.5(0.2)$ & $0.6(0.1)$ & .023 \\
\hline Remodeling index & $0.8(0.1)$ & $1.3(0.3)$ & $<.0001$ \\
\hline
\end{tabular}

a Variables are expressed as mean and SD.

Our findings emphasized that NR was almost as frequent as PR in symptomatic atherosclerotic MCA stenosis and NR lesions had less wall area and percentage of plaque burden. At reference sites, NR lesions had greater maximal wall thickness than PR lesions, with a trend toward significance; however, the difference may have no meaning, given the in-plane resolution. In addition, the remodeling pattern was associated with the eccentricity index, and PR lesions had a larger eccentricity index ( 0.6 versus $0.5, P=.023)$. These findings have potential implications for the mechanisms of arterial PR because it has been thought that in coronary atherosclerosis, PR mainly occurs as a consequence of the outward stretch of the vessel wall behind the plaque, as a direct response to the plaque release of metalloproteinases. ${ }^{1}$
Intracranial atherosclerotic stenosis is associated with a high risk of recurrent stroke, despite treatment with aspirin and standard management of vascular risk factors. Intracranial PTAS emerged as a valuable tool for patients refractory to medical therapy. ${ }^{16,17}$ In the PTAS procedure, the operators select the size of the balloon or stent on the basis of the reference vessel diameter during luminal imaging. However, for the patients with NR, sizing of the balloon may not take into account the diminished outer wall diameter and may increase the risk of vessel injury or even rupture when the balloon is inflated. Previous studies with coronary intervention have shown that patients with PR had the highest rate of major adverse cardiac events; in contrast, patients with NR may face high rates of in-hospital complications, including postinterventional dissection. ${ }^{2,3}$ The consequences of SAMMPRIS suggest that the remodeling pattern may also be a prominent factor that influences the rate of periprocedural complications. In future studies, we will use a wall imaging technique to study the remodeling pattern of other intracranial arteries (such as the ICA) and will assess the influence of local remodeling patterns on periprocedural complications in patients with intracranial PTAS.

Intracranial PTAS also showed that patients presenting with perforator ischemia may have an increased risk of perioperative stroke. ${ }^{6}$ PTAS restores the luminal diameter by pushing the plaque outward against the wall of the artery, which can cause plaque shifting, occluding the penetrating arteries. An eccentric plaque location on the superior and dorsal walls of the MCA, where most of perforators arise, may increase the risk of perforator occlusion. In this study, $34.1 \%$ of the lesions mainly involved the superior and dorsal MCA walls. These findings are meaningful. Patients with perforator syndromes were also included in SAMMPRIS. This feature may help explain the high risk of perioperative stroke. Patients with plaques near penetrating artery orifices may face a higher risk of perforator stroke. For those patients, a subsized balloon with low balloon inflation pressure may help reduce the risk of perforator stroke. ${ }^{18}$

We found that measurement reproducibility for vessel and luminal areas in the MCA was lower than the results of a previous study of the basilar artery. ${ }^{10}$ This finding seemed due to the confluence of the MCA with adjacent brain parenchyma, with little surrounding CSF reducing the conspicuity of its outer wall. By contrast, abundant CSF around the basilar artery may improve wall conspicuity. We obtained good measurement reproducibility with more CSF surrounding the MCA due to age-related brain involution, especially compared with the relatively young volunteers in a previous study. ${ }^{19}$

We excluded nearly half of the patients from final analysis in this study, with most cases related to obliquity of the scan plane and tortuous vessels. This choice caused a high exclusion rate and makes the current protocol inefficient for assessment of remodeling in clinical practice. Approaches to arterial wall imaging involving $3 \mathrm{D}$ acquisitions with short time durations are promising. ${ }^{19}$ These techniques, with the aid of multiple planar reconstruction, may help reduce the number of excluded cases and may be ideal for assessment of arterial remodeling in tortuous intracranial vessels.

Other limitations are as follows: First, values of vessel area and wall thickness may be overestimated. The overestimation may 
arise from lower in-plane and through-plane spatial resolution. This would lead to partial volume effects, resulting in an overestimation of the true vessel area and wall thickness, especially for reference sites. Another significant issue was that no effective technique for inflow and CSF suppression was applied in this study. Incomplete inflow or surrounding CSF suppression might mimic the vessel wall, causing an additional overestimation of the true vessel wall thickness. Second, although the proximal and distal minimally diseased vessels were also selected as reference sites in some cases, the number of lesions with negative remodeling may have been overestimated or underestimated due to remodeling of the reference sites in response to early-stage atherosclerosis.

\section{CONCLUSIONS}

2D high-resolution MR imaging can help assess the remodeling pattern and plaque distribution of MCA stenosis, but the imaging and postprocessing protocol for remodeling assessment needs to be improved in the tortuous course of the MCA or MCA ostium lesions or angled lesions due to artifacts and technical challenges related to obliquity.

Disclosures: Xin Lou—RELATED: Grant: National Natural Science Foundation of China (81101034), Comments: Contract grant sponsor: National Natural Science Foundation of China; Contract grant number: 81101034. Chunxue Wang-RELATED: Grant: National Science Foundation (grant No. 81071115), ${ }^{\star}$ UNRELATED: Board Membership: National Science Foundation (grant No. 81071115).* Wei-Jian Jiang-RELATED: Grant: National Natural Science Foundation of China, Comments: 2 grants (30670731, 81070925). *Money paid to the institution.

\section{REFERENCES}

1. Varnava AM. Relationship between coronary artery remodeling and plaque vulnerability. Circulation 2002;105:939-43

2. Wexberg P, Gyöngyösi M, Sperker W, et al. Pre-existing arterial remodeling is associated with in-hospital and late adverse cardiac events after coronary interventions in patients with stable angina pectoris. J Am Coll Cardiol 2000;36:1860-69

3. Yamagishi M, Terashima M, Awano K, et al. Morphology of vulnerable coronary plaque: insights from follow-up of patients examined by intravascular ultrasound before an acute coronary syndrome. J Am Coll Cardiol 2000;35:106-11

4. Aliabadi D, Tilli FV, Bowers TR, et al. Incidence and angiographic predictors of side branch occlusion following high-pressure intracoronary stenting. Am J Cardiol 1997;80:994-97
5. Chimowitz MI, Lynn MJ, Derdeyn CP, et al. Stenting versus aggressive medical therapy for intracranial arterial stenosis. $N$ Engl J Med 2011;365:993-1003

6. Abou-Chebl A, Steinmetz H. Critique of "Stenting versus aggressive medical therapy for intracranial arterial stenosis" by Chimowitz et al in the New England Journal of Medicine. Stroke 2012;43:616-20

7. Marks MP. Is there a future for endovascular treatment of intracranial atherosclerotic disease after Stenting and Aggressive Medical Management for Preventing Recurrent Stroke and Intracranial Stenosis (SAMMPRIS)? Stroke 2012;43:580-84

8. Lam WW, Wong KS, So NM, et al. Plaque volume measurement by magnetic resonance imaging as an index of remodeling of middle cerebral artery: correlation with transcranial color Doppler and magnetic resonance angiography. Cerebrovasc Dis 2004;17:166-69

9. Ryu CW, Jahng GH, Kim EJ, et al. High resolution wall and lumen MRI of the middle cerebral arteries at 3 Tesla. Cerebrovasc Dis 2009;27:433-42

10. Ma N, Jiang WJ, Lou X, et al. Arterial remodeling of advanced basilar atherosclerosis: a 3-Tesla MRI study. Neurology 2010;75:253-58

11. $\mathrm{Xu} \mathrm{WH}, \mathrm{Li} \mathrm{ML}, \mathrm{Gao}$, et al. In vivo high-resolution MR imaging of symptomatic and asymptomatic middle cerebral artery atherosclerotic stenosis. Atherosclerosis 2010;212:507-11

12. Klein IF, Lavallee PC, Touboul PJ, et al. In vivo middle cerebral artery plaque imaging by high-resolution MRI. Neurology 2006; 67:327-29

13. Underhill HR, Yarnykh VL, Hatsukami TS, et al. Carotid plaque morphology and composition: initial comparison between 1.5- and 3.0-T magnetic field strengths. Radiology 2008;248:550-60

14. Li F, McDermott M, Li D, et al. The association of lesion eccentricity with plaque morphology and components in the superficial femoral artery: a high-spatial-resolution, multi-contrast weighted CMR study. J Cardiovasc Magn Reson 2010;12:37

15. Xu WH, Li ML, Gao S, et al. Plaque distribution of stenotic middle cerebral artery and its clinical relevance. Stroke 2011;42:2957-59

16. Jiang WJ, Cheng-Ching E, Abou-Chebl A, et al. Multicenter analysis of stenting in symptomatic intracranial atherosclerosis. Neurosurgery 2012;70:25-30, discussion 31

17. Jiang WJ, Yu W, Du B, et al. Outcome of patients with $\geq 70 \%$ symptomatic intracranial stenosis after Wingspan stenting. Stroke 2011;42:1971-75

18. Jiang WJ, Yu W, Ma N, et al. High resolution MRI guided endovascular intervention of basilar artery disease. J NeuroInterv Surg 2011;3:375-78

19. Qiao Y, Steinman DA, Qin Q, et al. Intracranial arterial wall imaging using three-dimensional high isotropic resolution black blood MRI at 3.0 Tesla. J Magn Reson Imaging 2011;34:22-30 